\title{
Efficient scattering model of multi-layer systems with anisotropic films
}

\section{SNELL'S LAW FOR ANISOTROPIC MEDIA}

Snell's law, which amounts to phase continuity of waves at an interface, says that

$$
n_{\text {in }} \sin \theta_{\text {in }}=n_{\text {out }} \sin \theta_{\text {out }}
$$

for an incident wave seeing refractive index $n_{i n}$ and at an angle $\theta_{i n}$, and an outgoing wave (reflected or transmitted) seeing a refractive index $n_{\text {out }}$ and at an angle $\theta_{\text {out }}$. If the refractive indices of the waves are known (as in isotropic media), it is simple to find the angle of the outgoing waves. It is not so simple in the presence of anisotropic media, however, owing to the nature of the so-called extraordinary waves, who experience a direction dependent refractive index, i.e., $n_{\text {out }}=n_{\text {out }}\left(\theta_{\text {out }}\right)$.

To find the generalization of Snell's law to anisotropic media, we must consider the so-called wave normal surfaces. We do so using the coordinate system in figure 3 , where the plane of incidence is the $x z$-plane and the material boundary is the $z=0$ plane.

Wave solutions of Maxwell's equations in anisotropic media satisfy one of two conditions for the wave vector $k$. The first possibility is

$$
k_{x}^{2}=k_{y}^{2}=k_{z}^{2}=k_{0}^{2} n_{o}^{2}
$$

describing the ordinary wave. The wave normal surface is a sphere and the wave number $\left|\boldsymbol{k}_{o}\right|$ is always $k_{0} n_{0}$. Thus, the ordinary wave behaves exactly as a wave in isotropic media, and the direction of propagation is simple to find using Snell's law. The second possibility, describing the extraordinary wave, can be written as the quadratic form

$$
\boldsymbol{k}^{T} Q^{-1} \boldsymbol{k}=k_{0}^{2}
$$

where

$$
Q=\mathrm{R}^{T}\left(\begin{array}{ccc}
n_{e}^{2} & 0 & 0 \\
0 & n_{e}^{2} & 0 \\
0 & 0 & n_{o}^{2}
\end{array}\right) \mathrm{R}
$$

and $\mathrm{R}$ is the rotation matrix that maps the optical axis to the $z$-axis. The tangential component $k_{x}$ is fixed by the incident wave (and also $k_{y}=0$ ), and so the requirement that $k$ lies on the wave normal surface means equation S3 is a quadratic for $k_{z}$. Thus, for any given $k_{x}$, the two solutions of this quadratic give the directions of propagation of the forward and backward propagating extraordinary waves in the anisotropic layer.

With the directions of propagation of the waves fixed, the displacement fields of the waves can be found according to

$$
D_{o}=k_{o} \times c
$$

and

$$
D_{e}=k_{e} \times k_{e} \times c,
$$

where $\boldsymbol{k}_{o}$ and $\boldsymbol{k}_{e}$ are the wave vectors of the ordinary and extraordinary waves, $\boldsymbol{D}_{o}$ and $\boldsymbol{D}_{e}$ are their displacement fields, and $c$ is the optical axis. These relations also follow from Maxwell's equations. When the ordinary wave solution is chosen it forces the condition that $\boldsymbol{D}_{0}, \boldsymbol{k}_{0}$, and $c$ are all orthogonal. When the extraordinary wave solution is chosen it forces that $\boldsymbol{D}_{e}, \boldsymbol{k}_{e}$, and $c$ are all co-planar, with $\boldsymbol{D}_{e}$ and $\boldsymbol{k}_{e}$ orthogonal. More detail on the orientation of the electric fields can be found in many text books, e.g. [1]. 
The calculation of the Fresnel coefficients requires the normalized electric field orientations of the various waves inside the anisotropic medium. These are found by multiplying the $\boldsymbol{D}$ fields with inverse of the permittivity tensor

$$
\varepsilon=\mathrm{R}^{T}\left(\begin{array}{ccc}
n_{o}^{2} & 0 & 0 \\
0 & n_{o}^{2} & 0 \\
0 & 0 & n_{e}^{2}
\end{array}\right) \mathrm{R}
$$

and scaling appropriately.

\section{FRESNEL REFLECTION AND TRANSMISSION COEFFICIENTS AT THE INTERFACE BETWEEN ISOTROPIC AND ANISOTROPIC LAYERS}

The calculation of the Fresnel coefficients requires four different cases. The first two of these are where light is incident from an isotropic material onto an anisotropic material, for both s- and p-polarized light. The derivation is a small alteration to that of [2].

The material boundary is again taken to be the $z=0$ plane, and the plane of incidence to be the $x z$ plane. Since all waves must have the same time dependence, $e^{-i \omega t}$, this term is omitted, though it is implicitly understood to be present in all fields. Any wave present in the system can then be described as

$$
\boldsymbol{E}^{\alpha}=\hat{\boldsymbol{E}}^{\alpha} A_{\alpha} e^{i\left(K x+q_{\alpha} z\right)},
$$

where $\hat{\boldsymbol{E}}^{\alpha}$ is the polarization, $A_{\alpha}$ is the amplitude, $q_{\alpha}=\sqrt{k_{0}^{2} n_{\alpha}^{2}-K^{2}}$ is the normal wave vector component, and $n_{\alpha}$ is the refractive index seen by the $\alpha$ wave. This encompasses the forwards and backwards s- and p-waves in the isotropic medium, and the o- and e-waves in the anisotropic medium. By the Maxwell equation

$$
\nabla \times E=-\frac{\partial B}{\partial t}
$$

the corresponding magnetic fields are

$$
\boldsymbol{H}^{\alpha}=\frac{1}{i \mu_{0} \omega}\left(\begin{array}{c}
-\partial_{z} E_{y}^{\alpha} \\
\partial_{z} E_{x}^{\alpha}-i K E_{z}^{\alpha} \\
\partial_{x} E_{y}^{\alpha}
\end{array}\right) e^{i\left(K x+q_{\alpha} z\right)} .
$$

The electromagnetic boundary conditions that will be used are the continuity of the tangential components of $\boldsymbol{E}$ and $\boldsymbol{H}$, implying continuity of $E_{x}, E_{y}, \partial_{z} E_{y}$, and $\partial_{z} E_{x}-i K E_{z}$.

For the case that the incident field is s-polarized, the $z$ dependence of the incident field is given by

$$
\boldsymbol{E}^{i n}=\left(\begin{array}{l}
0 \\
1 \\
0
\end{array}\right) e^{i q_{i n} z}
$$

the reflected field as

$$
\boldsymbol{E}^{r}=r_{s S}\left(\begin{array}{c}
0 \\
1 \\
0
\end{array}\right) e^{-i q_{i n} z}-\frac{r_{s p}}{k_{i n}}\left(\begin{array}{c}
q_{i n} \\
0 \\
K
\end{array}\right) e^{-i q_{i n} z}
$$

and the transmitted field as

$$
\boldsymbol{E}^{t}=t_{s o} \hat{\boldsymbol{E}}^{o} e^{i q_{o} z}+t_{s e} \hat{\boldsymbol{E}}^{e} e^{i q_{e} z} .
$$

This defines the Fresnel coefficients $r_{s s}, r_{s p}, t_{s o}$, and $t_{s e}$ as the reflection and transmission of an s-wave to an s-, p-, o-, and e-wave, respectively. Applying the four continuity conditions to the fields on either side of the interface, $E^{i n}+E^{r}$ and $E^{t}$, and then solving the resulting four 
simultaneous equations gives

$$
\begin{aligned}
r_{s s} & =\left[\left(q_{i n}-q_{e}\right) A_{o} E_{y}^{e}-\left(q_{i n}-q_{o}\right) A_{e} E_{y}^{o}\right] D_{i n}^{-1}, \\
r_{s p} & =-2 k_{i n}\left(A_{o} E_{x}^{e}-A_{e} E_{x}^{o}\right) D_{i n}^{-1}, \\
t_{s o} & =-2 q_{i n} A_{e} D_{i n}^{-1}, \\
t_{s e} & =2 q_{i n} A_{o} D_{i n}^{-1},
\end{aligned}
$$

where the $A_{\alpha}$ terms are defined by

$$
A_{\alpha}=\left(k_{i n}^{2} q_{i n}^{-1}+q_{\alpha}\right) E_{x}^{\alpha}-K E_{z}^{\alpha},
$$

and the denominator $D_{\text {in }}$ by

$$
D_{\text {in }}=\left(q_{\text {in }}+q_{e}\right) A_{o} E_{y}^{e}-\left(q_{\text {in }}+q_{o}\right) A_{e} E_{y}^{o} .
$$

For an incident p-wave, we define the $z$ dependence of the incident field as

$$
\boldsymbol{E}^{i n}=\frac{1}{k_{\text {in }}}\left(\begin{array}{c}
q_{\text {in }} \\
0 \\
-K
\end{array}\right) e^{i q_{i n}}
$$

the reflected field as

$$
\boldsymbol{E}^{r}=r_{p s}\left(\begin{array}{c}
0 \\
1 \\
0
\end{array}\right) e^{-i q_{i n}}-\frac{r_{p p}}{k_{i n}}\left(\begin{array}{c}
q_{i n} \\
0 \\
K
\end{array}\right) e^{-i q_{i n}},
$$

and the transmitted field as

$$
\boldsymbol{E}^{t}=t_{p o} \hat{\boldsymbol{E}}^{o} e^{i q_{o} z}+t_{p e} \hat{\boldsymbol{E}}^{e} e^{i q_{e} z} .
$$

As before, applying the continuity conditions and solving the system of equations gives

$$
\begin{aligned}
r_{p p} & =-\frac{2 k_{i n}}{q_{i n}}\left[\left(q_{i n}+q_{e}\right) E_{y}^{e} E_{x}^{o}-\left(q_{i n}+q_{o}\right) E_{y}^{o} E_{x}^{e}\right] D^{-1}+1 \\
r_{p s} & =2 k_{i n}\left(q_{e}-q_{o}\right) E_{y}^{o} E_{y}^{e} D^{-1} \\
t_{p o} & =2 k_{i n}\left(q_{i n}+q_{e}\right) E_{y}^{e} D^{-1} \\
t_{p e} & =-2 k_{i n}\left(q_{i n}+q_{o}\right) E_{y}^{o} D^{-1} .
\end{aligned}
$$

It should be noted that these equation differ slightly from their derivation in [2]. Firstly, all terms in this work are determined in terms of the wavevector components $K$ and $q$, where they are originally determined in [2], in part, by an angle of propagation. The orientation of the coordinate axes and in which direction this angle is measured are ambiguous, but the problems arising from this can be avoided by writing the equations as above. This work also has a change of sign to [2] in the equations for $r_{p p}$ and $r_{s p}$. The author of [2] defines the p-polarization of the reflected wave as pointing to the left of the wave vector, while the convention used in this paper is that the positive orientation of the p-polarization is always to the right of the wave vector (as it is for the incident wave). This definition ensures a consistent definition of positive orientation, regardless of direction of travel, and avoids troubles leading to erroneous solutions when considering the sum of rays in multilayered systems.

The case of an o- or e-wave incident upon an isotropic material is now considered. This derivation is a small change upon that of [3], where the dependence on a propagation angle has been replaced with normal and tangential wave vector components. The forward and backward traveling waves are differentiated between with the superscripts + and - , respectively. In this way, the field $E^{o+}$ is the electric field of the forward traveling (incident) o-wave, while $q_{e-}$ is the normal component of the backward traveling (reflected) e-wave wave vector, etc.

Considering first an incident o-wave, the $z$ dependence of the incident field is

$$
\boldsymbol{E}^{i n}=\hat{\boldsymbol{E}}^{o+} e^{i q_{o+} z},
$$


the reflected field is

$$
\boldsymbol{E}^{r}=r_{o o} \hat{\boldsymbol{E}}^{o-} e^{i q_{o-} z}+r_{o e} \hat{\boldsymbol{E}}^{e-} e^{i q_{e-} z},
$$

and the transmitted field is

$$
\boldsymbol{E}^{t}=\frac{t_{\text {op }}}{k_{\text {out }}}\left(\begin{array}{c}
q_{\text {out }} \\
0 \\
-K
\end{array}\right) e^{i q_{\text {out }} z}+t_{\text {os }}\left(\begin{array}{l}
0 \\
1 \\
0
\end{array}\right) e^{i q_{\text {out }} z}
$$

The positive p-polarisation is again defined as lying to the right of the wavevector. Applying the boundary conditions and solving the system of equations then gives that

$$
\begin{aligned}
& r_{o o}=\left[B_{e-}\left(q_{o u t}-q_{o+}\right) E_{y}^{o+}-B_{o+}\left(q_{o u t}-q_{e-}\right) E_{y}^{e-}\right] D_{o u t}^{-1}, \\
& r_{o e}=\left[B_{o+}\left(q_{\text {out }}-q_{o-}\right) E_{y}^{o-}-B_{o-}\left(q_{\text {out }}-q_{o+}\right) E_{y}^{o+}\right] D_{o u t}^{-1}, \\
& t_{o s}=\left[B_{e-}\left(q_{o-}-q_{o+}\right) E_{y}^{o+} E_{y}^{o-}+B_{o-}\left(q_{o+}-q_{e-}\right) E_{y}^{o+} E_{y}^{e-}\right. \\
& \left.+B_{o+}\left(q_{e-}-q_{o-}\right) E_{y}^{o-} E_{y}^{e-}\right] D_{o u t}^{-1}, \\
& t_{\text {op }}=k_{\text {out }} q_{\text {out }}^{-1}\left\{B_{e-}\left[\left(q_{\text {out }}-q_{o+}\right) E_{x}^{o-} E_{y}^{o+}-\left(q_{\text {out }}-q_{o+}\right) E_{x}^{o+} E_{y}^{o-}\right]\right. \\
& +B_{o-}\left[\left(q_{o u t}-q_{e-}\right) E_{x}^{o+} E_{y}^{e-}-\left(q_{o u t}-q_{o+}\right) E_{x}^{e-} E_{y}^{o+}\right] \\
& \left.+B_{o+}\left[\left(q_{\text {out }}-q_{o-}\right) E_{x}^{e-} E_{y}^{o-}-\left(q_{\text {out }}-q_{e-}\right) E_{x}^{o-} E_{y}^{e-}\right]\right\} D_{\text {out }}^{-1},
\end{aligned}
$$

where the denominator is given by

$$
D_{\text {out }}=B_{o-}\left(q_{\text {out }}-q_{e-}\right) E_{y}^{e-}-B_{e-}\left(q_{\text {out }}-q_{o-}\right) E_{y}^{o-},
$$

and the $B_{\alpha}$ terms are given by

$$
B_{\alpha}=\left(k_{\text {out }}^{2} q_{\text {out }}^{-1}-q_{\alpha}\right) E_{x}^{\alpha}+K E_{z}^{\alpha}
$$

Repeating the same procedure for an incident e-wave, the $z$-dependence of the incident field is

$$
\boldsymbol{E}^{i n}=\hat{\boldsymbol{E}}^{e+} e^{i q_{e+} z},
$$

the reflected field is

$$
\boldsymbol{E}^{r}=r_{e o} \hat{\boldsymbol{E}}^{o-} e^{i q_{o-} z}+r_{e e} \hat{\boldsymbol{E}}^{e-} e^{i q_{e-} z},
$$

and the transmitted field as

$$
\boldsymbol{E}^{t}=\frac{t_{e p}}{k_{\text {out }}}\left(\begin{array}{c}
q_{\text {out }} \\
0 \\
-K
\end{array}\right) e^{i q_{\text {out }} z}+t_{e s}\left(\begin{array}{l}
0 \\
1 \\
0
\end{array}\right) e^{i q_{\text {out }} z}
$$

The Fresnel coefficients are then

$$
\begin{aligned}
r_{e o}= & {\left[B_{e-}\left(q_{\text {out }}-q_{e+}\right) E_{y}^{e+}-B_{e+}\left(q_{\text {out }}-q_{e-}\right) E_{y}^{e-}\right] D_{\text {out }}^{-1} } \\
r_{e e}= & {\left[B_{e+}\left(q_{\text {out }}-q_{o-}\right) E_{y}^{o-}-B_{o-}\left(q_{\text {out }}-q_{e+}\right) E_{y}^{e+}\right] D_{\text {out }}^{-1} } \\
t_{e s}= & {\left[B_{o-}\left(q_{e+}-q_{e-}\right) E_{y}^{e+} E_{y}^{e-}+B_{e-}\left(q_{o-}-q_{e+}\right) E_{y}^{e+} E_{y}^{o-}\right.} \\
& \left.+B_{e+}\left(q_{e-}-q_{o-}\right) E_{y}^{o-} E_{y}^{e-}\right] D_{\text {out }}^{-1} \\
t_{e p}= & k_{\text {out }} q_{\text {out }}^{-1}\left\{B_{o-}\left[\left(q_{\text {out }}-q_{e-}\right) E_{x}^{e+} E_{y}^{e-}-\left(q_{\text {out }}-q_{e+}\right) E_{x}^{e-} E_{y}^{e+}\right]\right. \\
& +B_{e-}\left[\left(q_{\text {out }}-q_{e+}\right) E_{x}^{o-} E_{y}^{e+}-\left(q_{\text {out }}-q_{o-}\right) E_{x}^{e+} E_{y}^{o-}\right] \\
& \left.+B_{e+}\left[\left(q_{\text {out }}-q_{o-}\right) E_{x}^{e-} E_{y}^{o-}-\left(q_{\text {out }}-q_{e-}\right) E_{x}^{o-} E_{y}^{e-}\right]\right\} D_{\text {out }}^{-1} .
\end{aligned}
$$




\section{CODES}

Matlab codes to reproduce the Iterated Ray Method traces in Figures 4 and 5 are available from Code 1, [4].

\section{REFERENCES}

1. K. Iizuka, Elements of Photonics, Volume I: In Free Space and Special Media, vol. 41 (John Wiley \& Sons, 2002).

2. J. Lekner, "Reflection and refraction by uniaxial crystals," J. Physics: Condens. Matter 3, 6121 (1991).

3. E. S. Thomson, L. A. Wilen, and J. S. Wettlaufer, "Light scattering from an isotropic layer between uniaxial crystals," J. Physics: Condens. Matter 21, 195407 (2009).

4. J. R. Gill, E. Perivolari, M. Kaczmarek, and G. D'Alessandro, "Matlab implementation of the iterated ray method algorithm," (2021), https:/ / doi.org/10.5258/SOTON/D1779. 